\title{
The role of extracellular biophysical cues in modulating the Hippo-YAP pathway
}

\author{
Jung-Soon $\mathrm{Mo}^{*}$ \\ Genomic Instability Research Center (GIRC), Ajou University School of Medicine, Suwon 16499, Korea
}

\begin{abstract}
The Hippo signaling pathway plays an essential role in adult-tissue homeostasis and organ-size control. In Drosophila and vertebrates, it consists of a highly conserved kinase cascade, which involves MST and Lats that negatively regulate the activity of the downstream transcription coactivators, YAP and TAZ. By interacting with TEADs and other transcription factors, they mediate both proliferative and antiapoptotic gene expression and thus regulate tissue repair and regeneration. Dysregulation or mutation of the Hippo pathway is linked to tumorigenesis and cancer development. Recent studies have uncovered multiple upstream inputs, including cell density, mechanical stress, G-protein-coupled receptor (GPCR) signaling, and nutrients, that modulate Hippo pathway activity. This review focuses on the role of the Hippo pathway as effector of these biophysical cues and its potential implications in tissue homeostasis and cancer. [BMB Reports 2017; 50(2): 71-78]
\end{abstract}

\section{INTRODUCTION}

The size of each organ is determined by cell number and cell size. This process involves many signaling pathways during development, and regeneration controls cell number in tissue and organs. In recent years, the Hippo tumor-suppressor pathway has emerged as a key regulator of organ size and tumorigenesis by inhibiting cell proliferation, promoting apoptosis, and limiting stem/progenitor-cell expansion (1). This pathway was initially identified by means of genetic mosaic screens for growth-control genes. In Drosophila, the core components of the Hippo pathway include a kinase cascade of Ste20-like kinase Hippo (Hpo), with the scaffolding protein Salvador (Sav), and NDR family kinase Warts (Wts), with its regulatory protein Mob as Tumor Suppressor (Mats) (2-8). Hpo forms a complex with Sav to phosphorylate and activate Wts,

*Corresponding author. Tel: +82-31-219-7803; Fax: +82-31-2197802; E-mail: j5mo@ajou.ac.kr

https://doi.org/10.5483/BMBRep.2017.50.2.199

Received 23 November 2016

Keywords: YAP, TAZ, Hippo pathway, Mechanical cues, GPCR, Nutrient which then interacts with Mats (9-11). Wts directly phosphorylates the transcriptional co-activator Yki (Yorkie), promoting its interaction with 14-3-3 and leading to YAP cytoplasmic retention (12-16). Inactivation of the Hippo pathway reduces its downstream kinase-mediated YAP phosphorylation. The unphosphorylated YAP translocates to the nucleus, where it binds with the TEAD/TEF- family transcription factor Sd (Scalloped) to activate transcription of target genes, promoting cell survival and proliferation $(17,18)$. Then the pathway and its cellular functions, including cell survival, proliferation, and organ-size control is evolutionally conserved in mammals $(13,19)$. Core components of the mammalian Hippo pathway include a kinase cascade of mammalian STE20-like protein kinase $1 / 2$ (MST1/2) and the large tumor suppressor $1 / 2$ (Lats1/2). MST1/2 in complex with its regulatory protein Sav1 phosphorylates and activates Lats $1 / 2$ kinases, which also form a complex with its regulatory protein, Mob1. The Yes-associated protein (YAP) is a transcription co-activator and an important downstream effector of the Hippo pathway. YAP was first identified as a non-receptor tyrosine kinase YES1 binding partner (20). The physiological importance of YAP/TAZ was uncovered after the identification of Drosophila Yki as a key effector of the Hippo pathway (12). In a detailed study of Hippo kinase cascade, the Hippo pathway kinase Lats $1 / 2$ inhibits YAP by direct phosphorylation of five consensus HXRXXS motifs (13, 19, 21-23). Phosphorylation of S127 in YAP results in cytoplasmic sequestration via 14-3-3 binding and therefore inactivates YAP. Thus YAP is degraded by the proteasome in a ubiquitin-dependent manner following phosphorylation of Ser 397. A transcriptional coactivator with PDZ-binding motif (TAZ, also called WWTR1), a paralog of YAP in mammals, was initially identified as a 14-3-3 binding protein in a phosphorylation-dependent manner (24). TAZ contains four consensus Lats $1 / 2$ target motifs and is similarly regulated by Lats $1 / 2(23,25)$. Conversely, unphosphorylated YAP localizes in the nucleus and acts mainly through the TEAD family transcription factors to stimulate expression of genes that promote proliferation and inhibit apoptosis $(26,27)$. Besides TEADs, YAP/TAZ can also interact with several different transcription factors, including Smad, Runx1/2, p73, ErbB4, Pax3, and T-box transcription factor 5 (TBX5) to mediate transcription and a diverse array of cellular functions (28). 


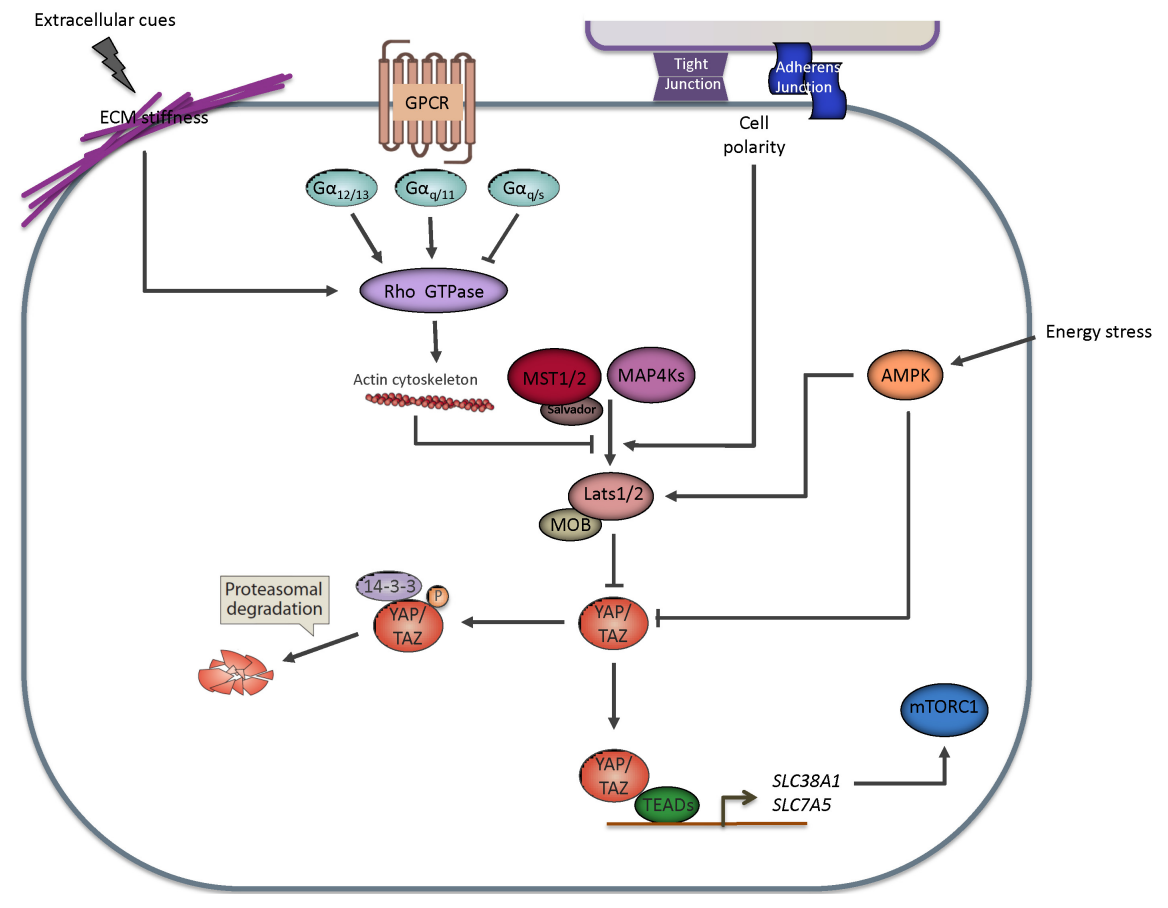

Fig. 1. Regulation of the Hippo-YAP pathway by extracellular biophysical cues. Mechanical stress inhibits Lats $1 / 2$ kinase activity via Rho GTPase and the actin cytoskeleton. GPCR signaling can either activate or inhibit YAP/TAZ activity through the coupled $\mathrm{G} \alpha$ protein. Cellular junction and cell polarity modulate the Hippo pathway. Nutrient signaling modulates the core Hippo kinase and YAP activity through AMPK. YAP/TAZ activity is involved in amino-acid induced mTORC1 activation.
In recent years, beyond the main components of the Hippo pathway defined above, many other additional regulators have been found to regulate the Hippo pathway. Accumulating evidence suggests that the core Hippo kinase cascade and YAP/TAZ incorporate various upstream responses, enabling dynamic regulation of tissue homeostasis and cancer (29). In this review we will focus on the expanding roles of YAP/TAZ as mediators of responses to biophysical cues, especially mechanical stress, GPCR signaling, and nutrient signaling (Fig. 1).

\section{REGULATION OF HIPPO-YAP PATHWAY BY EXTRACELLULAR MECHANICAL CUES}

Growth and development is the net result of various harmonized events of cells to adjust to physical restraints and extracellular mechanical signals. For instance, the cell-densitymediated cell-cell contact causes a growth-inhibitory signaling pathway that in large part is mediated by the Hippo pathway $(19,30,31)$. Abundant cell-cell contact activates Lats and inactivates YAP which is critically important for contact inhibition. The regulation of YAP/TAZ-TEAD mediated transcription in response to contact inhibition is also essential for embryo development (32). In addition, the apical-basal cell polarity protein, adherens junctions, and tight junctions provide the intrinsic cues to regulate Lats $1 / 2$ and restrict YAP activity (33). Interestingly, it was found that YAP /TAZ activity and subcellular localization are regulated by extracellular matrix (ECM) stiffness. When cells are cultured on stiff ECM, YAP/TAZ predominantly localizes to nuclei and promotes
YAP/TAZ transcriptional activity. However, when cells are cultured on soft ECM, cells are round and adhesion with ECM is limited. Likewise, YAP/TAZ activity and subcellular localization depend on the adhesive area. Furthermore, YAP/TAZ activity is modulated by cell stretching, spreading, and cell size through changes in the cytoskeleton (34-36). More importantly, activation of YAP/TAZ by rigidity of the extracellular matrix greatly improves differentiation of human pluripotent stem cells in motor neurons (37).

Morphological manipulation and stress-fiber quantity changes in response to physical forces inhibit the Hippo pathway and promote nuclear YAP localization in a way similar to matrix stiffness (38). Also, induction of F-actin polymerization by loss of capping proteins, Cpa and $\mathrm{Cpb}$, or overexpressing an activated actin nucleation factor Diaphanous, leads to cell proliferation and overgrowth in imaginal discs. Studies on Drosophila have demonstrated that changing F-actin levels correlates with activation of $\mathrm{Yki}$ and causes overgrowth (39). In contrast, reduction of actin-capping protein or inhibition of Capulet, which all induce abnormal F-actin polymerization, sustains Hippo pathway activity, thereby inducing expression of Yki target genes near the apical surface in Drosophila (40). The outcome of F-actin in regulation of YAP is also likely evolutionarily conserved in mammals, since deletion of the destrin gene, an actin-depolymerizing factor, increases the aberrant actin cytoskeleton and leads to epithelial hyperproliferation (41). This was further established by the observation that CapZ or Cofilin restricts YAP nuclear localization and YAP transcriptional activity (35). The structure of actin 
cytoskeleton is responsible for the transduction of mechanical stress in cells. The Rho GTPases, which have great effects on actin cytoskeleton organization, is a crucial regulator of YAP/TAZ activity. For example, disruption of F-actin or inhibition of Rho by specific inhibitors considerably reduces YAP nuclear translocation and activity $(36,38,42)$. The molecular mechanism of YAP/TAZ regulation by actin cytoskeleton and mechanical stress has not yet been fully understood. Previous studies ignore MST1/2 and Lats1/2 in the regulation of YAP/TAZ nuclear translocation and transcriptional activation, because knockdown of Lats $1 / 2$ is not enough to recover YAP/TAZ activity by ECM stiffness (36). However, under detached conditions, the Lats $1 / 2$ leads to YAP inhibition in a cytoskeletondependent manner (42). Similar to that observed in cell detachment, mechanical strain lead to Lats $1 / 2$ inhibition to activate YAP in a JNK-dependent manner (43). Accordingly, it is possible that both Lats1/2-dependent and -independent mechanisms are included in the YAP/TAZ regulation by mechanical stress. Recent findings have implied that YAP/TAZ plays a role in breast-cancer development in response to mechanical stress. For instance, many cancers such as breast cancer have elevated extracellular stiffness accompanied by a changed ECM composition compared with that of normal mammary tissue. Remarkably, it was shown that YAP is activated in cancer-associated fibroblasts (CAFs), and that its function is required for matrix stiffing (44). Higher extracellular stiffness affects YAP activity and hence contributes to the tumor microenvironment. It was proposed that YAP conditioned the tumor microenvironment by modulating matrix stiffening and production of YAP/TAZ target genes, such as AREG, CYR61, and CTGF, to promote tumorigenesis. TAZ is shown to be upregulated in high-grade and metastatic breast tumors (45). In addition, TAZ confers cancer stem-cell traits on breast cancer cells, and cancer stem cells showing high levels of TAZ are observed in high-grade tumors (46). The YAP/TAZ activity and the extracellular matrix provide a positive feedback mechanism, in which cancer cells promote matrix stiffening that further activates YAP/TAZ as transcriptional co-activators. Recent studies also show that disturbed flow activates YAP/ TAZ target-gene expression through the modulation of RhoGTPase activities, demonstrating a significant role for YAP/TAZ in mediating mechanical cues and vascular homeostasis (47-49).

Overall, many studies have suggested that actin RhoGTPases serves as a sensor to connect mechanical cues to YAP/TAZ activity. However, the involvement of the Hippo pathway kinases MST1/2 and Lats $1 / 2$ are not completely understood. Future studies are required to define the mechanotransducers as YAP/TAZ effectors as well as the role of the core Hippo kinase cascade in regulation of YAP/TAZ by mechanical cues.

\section{REGULATION OF THE HIPPO-YAP PATHWAY BY CELL-SURFACE RECEPTORS AND SOLUBLE MOLECULES}

Under normal physiological conditions, hormones are chemical messengers that stimulate cell growth and proliferation. Such molecules are released from the cell sending the signal, cross over the gap between cells by diffusion, and interact with specific receptors in another cell, triggering a response in that cell by activating intracellular signaling which leads to physiological changes inside the cell. Physiological changes that result from soluble molecules tightly regulate cell growth, proliferation, and differentiation. It has been hypothesized that the extracellular environment, such as hormones, might regulate tissue growth and homeostasis through cell-surface receptors and Hippo pathway components. An important discovery came with the demonstration that diffusive lipid molecules, such as lysophosphatidic acid (LPA) and sphingosine1-phosphate (S1P), could trigger intracellular signaling cascades and activate YAP/TAZ through their cognate $G$ proteincoupled receptors (GPCRs) $(50,51)$. Additional study confirmed that both LPA1 and LPA3 are involved in LPA-induced YAP/TAZ activation, which is likely to be relate to long-term cell migration; PP1A is required for the LPA-YAP effects in epithelial ovarian cancer cells (52). Consistent with the roles of LPA and S1P in regulating YAP/TAZ, thrombin, the ligand of protease-activated receptors (PARs), stimulated YAP/TAZ activities by inducing its dephosphorylation and target-genes expression (53). GPCRs recognize numerous extracellular signals and transduce them to heterotrimeric G proteins, which further transduce these intracellular signals to appropriate downstream effectors and thereby play a main role in various signaling pathways (54). Mechanistically, LPA, S1P, and thrombin counteract $\mathrm{G}_{12 / 13^{-}}$and $\mathrm{G} \alpha_{\mathrm{q} / 11^{-}}$-coupled GPCRs to activate Rho-GTPases. Activation of Rho-GTPase serves as a key mediator in the activation of YAP/TAZ from upstream GPCRs. YAP/TAZ activity could be either activated or inhibited, depending on the $G$ protein coupled to the GPCRs. Activation of Gos-coupled GPCRs by epinephrine and glucagon increases Lats $1 / 2$ kinase activities and inactivates YAP/TAZ in a manner dependent on protein kinase A (PKA) (55). Hence, depending on the kind of G proteins, GPCRs can differentially regulate Lats $1 / 2$ to stimulate or suppress YAP activity. Other studies further demonstrate that the core Hippo kinase cascade and YAP/TAZ activity are regulated by GPCRs in response to various hormonal cues. For instance, GPR68, a proton-sensing GPCR, is activated in response to a decrease in extracellular $\mathrm{pH}$ and is required for the $\mathrm{pH}$-dependent regulation of the proliferation and apoptosis. Under a decrease in extracellular $\mathrm{pH}$, GPR68 leads to an increase in the proliferation and a decrease in apoptosis of cells with abundant proton-sensing GPCR expression. In addition, it was found that YAP functions

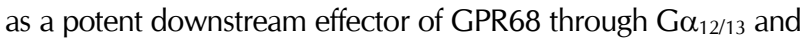
Rho GTPase $(56,57)$. Besides, YAP is required for the $\mathrm{pH}$ - 
dependent regulation of the differentiation of mesenchymal stem cells (MSCs) into cancer-associated fibroblasts, CAFs. Furthermore, stimulation of the G-protein-coupled estrogen receptor (GPER) by estrogen activates YAP/TAZ and regulates the expression of numerous genes, including well-characterized target genes via the $\mathrm{G} \alpha_{\mathrm{q} / 11}, \mathrm{PLC} \beta / \mathrm{PKC}$, and Rho/Rock signaling pathways. It was proposed that TAZ was required for breast cancer cell proliferation, migration, and tumor growth. As expected, TAZ expression positively correlated with GPER expression in human invasive ductal carcinoma (IDC) specimens, indicating that YAP/TAZ may be activated by estrogen in breast cancer (58). TxA2 exerts its biological activity through its cognate thromboxane A2 receptor (TP) receptor that couples with $G \alpha_{q / 11}, G \alpha_{12 / 13}$, and other trimeric $G$ proteins to regulate downstream effectors. TP has been implicated in promoting cell migration and proliferation of vascular smooth muscle cells (VSMCs). Treatment of the cells with thromboxane A2 (TP) activation promotes DNA synthesis and induces VSMC proliferation and migration in a manner dependent on YAP/TAZ (59). Thromboxane A2 signaling increases YAP/TAZ activity in VSMCs and other cell types via $\mathrm{G} \alpha_{12 / 13}$, providing YAP/TAZ as potential therapeutic target for VSMC-mediated vascular disease. This study shows for the first time that Angll binding to the angiotensin II type 1 receptor (AT1R) can inhibit the Hippo pathway and activate YAP (60). As GPCR's coupling to the $G$ protein subclass $G \alpha_{q} / 11$, in general, are able to activate YAP, we therefore expected the same influence from the AT1R, which mainly couples to $G \alpha_{q / 11}$. Stimulation of the AT1R with Angll showed decreased Lats $1 / 2$ activation, which was accompanied by decreased phosphorylation of its target YAP in HEK293T cells. Despite the initial observation of Angll as a stimulant of YAP dephosphorylation and nuclear localization, the Hippo pathway is not activated by stimulation with Angll in podocytes, which show a deactivated pathway. However, the actin cytoskeleton disruption with Latrunculin B reactivates Lats $1 / 2$ kinase activity, resulting in increased cytoplasmic YAP localization accompanied by a strong induction of apoptosis. Angiotensin II receptor serves as an upstream regulator of the Hippo pathway. The control of Lats $1 / 2$ activation and subsequent YAP localization is important for podocyte homeostasis and survival.

In addition to GPCRs, several other morphogenic factors elicit diverse receptor-mediated signaling pathways to control development and tissue homeostasis. The cytokine receptor leukemia inhibitory factor receptor (LIFR) activates the Hippo kinase cascade (61). The PI3K-PDK1 pathway disrupts the core Hippo complex in response to EGF, leading to inactivation of Lats1/2 and activation of YAP (62). Furthermore, YAP/TAZ is a critical mediator of the canonical $\mathrm{Wnt} / \mathrm{b}$-catenin and noncanonical alternative Wnt signaling. Two independent groups revealed that Wnt ligands could activate YAP/TAZ through their corresponding GPCRs, frizzled (FZD) receptors, although distinct signaling mechanisms are utilized (63-69). In the present studies, TGF $\beta$ and bone morphogenetic protein (BMP) sustain YAP/TAZ activity. Interaction between TAZ and TGF $\beta$ regulated SMAD2 and SMAD3 governs their nuclear localization and target-genes expression. YAP can also be involved with SMAD1 and synergize transcriptional activation of BMP signaling (70-72).

GPCRs are the superfamily of the cell-surface receptors mediating the actions of hundreds of extracellular molecules that have a pivotal role in many physiological functions and in multiple diseases, including the development of cancer and cancer metastasis (54). Elevated expression of GPCRs or activating mutation of $\mathrm{G} \alpha$ leads to aberrant YAP activation and has been found in several types of cancers $(58,73-75)$. The regulation of YAP/TAZ by GPCRs implies that the Hippo pathway not only is modulated by many extracellular signals and cell-surface receptors, but also contributes to a wide range of physiological regulation and may function as the key mediator of GPCR agonists or antagonists for disease progression.

\section{REGULATION OF THE HIPPO-YAP PATHWAY BY NUTRIENT SIGNALING}

Nutrients and energy metabolism such as glucose, amino acids, and fatty acids are building blocks of the cells that promote cell growth. Glucose is an abundant fuel and the most widely used as an energy source in living organisms. Therefore, it is anticipated that nutrient signals can modulate YAP and TAZ activities. As expected, deprived of glucose, AMPK directly phosphorylates S793 of AMOLT1 and increases AMOTL1 protein levels, resulting in YAP inhibition in a Lats $1 / 2$ dependent manner (76). Furthermore, energy stressactivated AMPK directly phosphorylates YAP at multiple sites, and this phosphorylation interferes with the interaction between YAP and TEAD, thus contributing to its inactivation and inhibition of TEAD-mediated transcription $(77,78)$. LKB/ STK11 is a known tumor suppressor and a major upstream regulator of AMPK. LKB1 represses YAP activity via either the core Hippo kinase cascade dependent or independent pathway $(79,80)$. On the other hand, loss of LKB1 and AMPK contributes to Yki activation and accelerated proliferation in the Drosophila (81). LKB1-mediated inhibition of Yki activity is mediated by AMPK and is independent of the $\mathrm{Hpo} / \mathrm{Wts}$ kinase cascade, suggesting a potential energy-dependent pathway controlling proliferation in the central brain $(\mathrm{CB})$ and ventral nerve-cord developmental neural systems (VNC).

Additionally, the Hippo pathway also responds to nutrients other than glucose. YAP/TAZ potentiates mTORC activity by increasing expression of the high-affinity L-type amino-acid transporter (LAT1), which is a heterodimer of SLC7A5 and SLC3A2. YAP/TAZ and TEAD directly induce transcription of LSC7A5, which rescues SLC3A2 protein expression by dimer formation, to increase LAT1 expression and amino-acid uptake $(82,83)$. In parallel, mTOR also is a master regulator of cellular growth and survival and stimulates cellular metabolic processes, such as protein synthesis. An mTORC signaling 
pathway is reported to drive YAP activation and its targetgenes expression in perivascular epithelioid cell tumors and glioblastomas (84-86). Both outputs of TOR are required for wing cells to divide and gain mass under Yki-Sd control in Drosophila (87). Previous evidence indicated that YAP, a main target of inhibition by the Hippo pathway, can activate AKT through miR-20-mediated inhibition of PTEN (88). These data, combined with a recent study, indicated that mTORC2 can regulate AKT activity, both directly and indirectly through inhibition of the Hippo pathway and activation of YAP (85). In addition, AKT and MST1 were previously shown to mutually inhibit each other $(89,90)$. Thus, mTOR2 and the Hippo pathway can engage in crosstalk at multiple levels. Of note, mTORC2 was also shown to activate SGK1 and PRKCA/PKC $\alpha$ (91-93). Besides lowering the cellular cholesterol levels, inhibition of the mevalonate pathway inhibits YAP/TAZ nuclear localization and transcriptional response, possibly because of inhibition of the Rho GTPases, which require a complex network by which cytoskeleton impinges on YAP/TAZ activation (94). In addition, other nutrients have been shown to be important in regulation of the Hippo pathway. For instance, the salt-induced kinases have been implicated in nutrient sensing that promotes Yki target-gene expression and tissue overgrowth through phosphorylation of Sav at Ser413 (95).

The most recognized functional output of YAP and TAZ is to promote cell survival and proliferation by cellular nutrient status. Therefore, given the central role of the Hippo signaling pathway in nutrient sensing, understanding how nutrients contribute to cancer development remains an area of intense investigation.

\section{CONCLUSIONS}

Extensive research within recent decades has identified more components and other signaling pathways linked with the Hippo pathway and YAP/TAZ regulation, since many core Hippo-pathway components have been discovered in Drosophila and mammals. In recent years, the Hippo pathway has been influentially and intensely regulated by a wide array of extracellular biophysical cues, including mechanical cues, cell-surface receptors, and nutrient signaling from neighboring cells and the extracellular matrix. The core Hippo kinase cascade integrates multiple upstream inputs to control YAP/TAZ activity, allowing vigorous regulation of cellular processes, such as proliferation, differentiation, and apoptosis in intricate physiological contexts and in cancer.

However, it is important to realize that gaps still remain in understanding the key molecular mechanisms in extracellular biophysical cues. For example, it is unclear whether Lats $1 / 2$ kinase is involved in YAP/TAZ regulation by actin cytoskeleton under mechanical cues. Current evidence showed that Lats $1 / 2$ kinase activity is important for GPCR-mediated YAP/TAZ regulation, but Mst1/2 is not required for YAP/TAZ regulation by both mechanical cues and GPCR signaling. This suggests that other mechanisms or other unknown molecules may be involved in the process in response to the physiological environment. Furthermore, the detailed mechanism by which the actin cytoskeleton transmits upstream cues to modulate Lats $1 / 2$ kinase activity has yet to be uncovered. The possibly existing Lats $1 / 2$-independent mechanism of YAP/TAZ regulation by the actin cytoskeleton also has yet to be uncovered. It will also be interesting to define how YAP/TAZ may converge on these mechanical and hormonal cues to respond to the environment in an appropriate manner. For example, both mechanical cues and cell-surface receptors, especially GPCRs, signal input into regulation of Rho GTPase activity and thus affect YAP/TAZ activity.

Taken together, the YAP/TAZ are unquestionably important mediators of extracellular biophysical cues in regulation of organ size control, regeneration, and tumorigenesis, and thus would be legitimate attractive potential therapeutic targets for cancer therapy.

\section{ACKNOWLEDGEMENTS}

This research was supported by Basic Science Research Program through the National Research Foundation of Korea (NRF) funded by the Ministry of Science, Ict and Future Planning [NRF-2016R1C1B2016135 (Young Researcher Program), No.2011-0030043 (SRC)] and the New Faculty Research Fund of Ajou University School of Medicine.

\section{CONFLICTS OF INTEREST}

The authors have no conflicting financial interests.

\section{REFERENCES}

1. Yu FX, Zhao B and Guan KL (2015) Hippo Pathway in Organ Size Control. Tissue Homeostasis, and Cancer. Cell $163,811-828$

2. Harvey KF, Pfleger CM and Hariharan IK (2003) The Drosophila Mst ortholog, hippo, restricts growth and cell proliferation and promotes apoptosis. Cell 114, 457-467

3. Jia J, Zhang W, Wang B, Trinko R and Jiang J (2003) The Drosophila Ste20 family kinase dMST functions as a tumor suppressor by restricting cell proliferation and promoting apoptosis. Genes Dev 17, 2514-2519

4. Justice RW, Zilian O, Woods DF, Noll M and Bryant PJ (1995) The Drosophila tumor suppressor gene warts encodes a homolog of human myotonic dystrophy kinase and is required for the control of cell shape and proliferation. Genes Dev 9, 534-546

5. Pantalacci S, Tapon N and Leopold P (2003) The Salvador partner Hippo promotes apoptosis and cell-cycle exit in Drosophila. Nat Cell Biol 5, 921-927

6. Xu T, Wang W, Zhang S, Stewart RA and Yu W (1995) Identifying tumor suppressors in genetic mosaics: the Drosophila lats gene encodes a putative protein kinase. Development 121, 1053-1063 
7. Udan RS, Kango-Singh M, Nolo R, Tao C and Halder G (2003) Hippo promotes proliferation arrest and apoptosis in the Salvador/Warts pathway. Nat Cell Biol 5, 914-920

8. Wu S, Huang J, Dong J and Pan D (2003) Hippo encodes a Ste-20 family protein kinase that restricts cell proliferation and promotes apoptosis in conjunction with salvador and warts. Cell 114, 445-456

9. Kango-Singh M, Nolo R, Tao C et al (2002) Shar-pei mediates cell proliferation arrest during imaginal disc growth in Drosophila. Development 129, 5719-5730

10. Tapon N, Harvey KF, Bell DW et al (2002) Salvador promotes both cell cycle exit and apoptosis in Drosophila and is mutated in human cancer cell lines. Cell 110, 467-478

11. Lai ZC, Wei X, Shimizu T et al (2005) Control of cell proliferation and apoptosis by mob as tumor suppressor, mats. Cell 120, 675-685

12. Huang J, Wu S, Barrera J, Matthews K and Pan D (2005) The Hippo signaling pathway coordinately regulates cell proliferation and apoptosis by inactivating Yorkie, the Drosophila homolog of YAP. Cell 122, 421-434

13. Dong J, Feldmann G, Huang J et al (2007) Elucidation of a universal size-control mechanism in Drosophila and mammals. Cell 130, 1120-1133

14. Staley BK and Irvine KD (2012) Hippo signaling in Drosophila: recent advances and insights. Dev Dyn 241, 3-15

15. Oh $\mathrm{H}$ and Irvine KD (2008) In vivo regulation of Yorkie phosphorylation and localization. Development 135, 1081-1088

16. Ren F, Zhang L and Jiang J (2010) Hippo signaling regulates Yorkie nuclear localization and activity through 14-3-3 dependent and independent mechanisms. Dev Biol 337, 303-312

17. Mahoney WM Jr, Hong JH, Yaffe MB and Farrance IK (2005) The transcriptional co-activator TAZ interacts differentially with transcriptional enhancer factor-1 (TEF-1) family members. Biochem J 388, 217-225

18. Vassilev A, Kaneko KJ, Shu H, Zhao Y and DePamphilis ML (2001) TEAD/TEF transcription factors utilize the activation domain of YAP65, a Src/Yes-associated protein localized in the cytoplasm. Genes Dev 15, 1229-1241

19. Zhao B, Wei X, Li W et al (2007) Inactivation of YAP oncoprotein by the Hippo pathway is involved in cell contact inhibition and tissue growth control. Genes Dev 21, 2747-2761

20. Sudol M (1994) Yes-associated protein (YAP65) is a proline-rich phosphoprotein that binds to the $\mathrm{SH} 3$ domain of the Yes proto-oncogene product. Oncogene 9, 21452152

21. Hao Y, Chun A, Cheung K, Rashidi B and Yang X (2008) Tumor suppressor LATS1 is a negative regulator of oncogene YAP. J Biol Chem 283, 5496-5509

22. Oka T, Mazack V and Sudol M (2008) Mst2 and Lats kinases regulate apoptotic function of Yes kinaseassociated protein (YAP). J Biol Chem 283, 27534-27546

23. Lei QY, Zhang H, Zhao B et al (2008) TAZ promotes cell proliferation and epithelial-mesenchymal transition and is inhibited by the Hippo pathway. Mol Cell Biol 28, 2426-2436
24. Kanai F, Marignani PA, Sarbassova D et al (2000) TAZ: a novel transcriptional co-activator regulated by interactions with 14-3-3 and PDZ domain proteins. EMBO J 19, 6778-6791

25. Liu CY, Zha ZY, Zhou X et al (2010) The Hippo tumor pathway promotes TAZ degradation by phosphorylating a phosphodegron and recruiting the SCF $\{$ beta\}-TrCP E3 ligase. J Biol Chem 285, 37159-37169

26. Zhao B, Ye X, Yu J et al (2008) TEAD mediates YAPdependent gene induction and growth control. Genes Dev 22, 1962-1971

27. Zhang H, Liu CY, Zha ZY et al (2009) TEAD transcription factors mediate the function of TAZ in cell growth and epithelial-mesenchymal transition. J Biol Chem 284, 13355-13362

28. Zhu C, Li L and Zhao B (2015) The regulation and function of YAP transcription co-activator. Acta Biochim Biophys Sin (Shanghai) 47, 16-28

29. Zanconato F, Cordenonsi M and Piccolo S (2016) YAP/TAZ at the roots of cancer. Cancer Cell 29, 783-803

30. Ota M and Sasaki H (2008) Mammalian Tead proteins regulate cell proliferation and contact inhibition as transcriptional mediators of Hippo signaling. Development $135,4059-4069$

31. Nishioka N, Inoue K, Adachi K et al (2009) The Hippo signaling pathway components Lats and Yap pattern Tead4 activity to distinguish mouse trophectoderm from inner cell mass. Dev Cell 16, 398-410

32. Gumbiner BM and Kim NG (2014) The Hippo-YAP signaling pathway and contact inhibition of growth. J Cell Sci 127, 709-717

33. Yang CC, Graves HK, Moya IM et al (2015) Differential regulation of the Hippo pathway by adherens junctions and apical-basal cell polarity modules. Proc Natl Acad Sci U S A 112, 1785-1790

34. Driscoll TP, Cosgrove BD, Heo SJ, Shurden ZE and Mauck RL (2015) Cytoskeletal to Nuclear Strain Transfer Regulates YAP Signaling in Mesenchymal Stem Cells. Biophys J 108, 2783-2793

35. Aragona M, Panciera T, Manfrin A et al (2013) A mechanical checkpoint controls multicellular growth through YAP/TAZ regulation by actin-processing factors. Cell 154, 1047-1059

36. Dupont S, Morsut L, Aragona M et al (2011) Role of YAP/TAZ in mechanotransduction. Nature 474, 179-183

37. Sun Y, Yong KM, Villa-Diaz LG et al (2014) Hippo/YAPmediated rigidity-dependent motor neuron differentiation of human pluripotent stem cells. Nat Mater 13, 599-604

38. Wada K, Itoga K, Okano T, Yonemura S and Sasaki H (2011) Hippo pathway regulation by cell morphology and stress fibers. Development 138, 3907-3914

39. Sansores-Garcia L, Bossuyt W, Wada K et al (2011) Modulating F-actin organization induces organ growth by affecting the Hippo pathway. EMBO J 30, 2325-2335

40. Fernandez BG, Gaspar P, Bras-Pereira C, Jezowska B, Rebelo SR and Janody F (2011) Actin-Capping Protein and the Hippo pathway regulate F-actin and tissue growth in Drosophila. Development 138, 2337-2346

41. Ikeda S, Cunningham LA, Boggess D et al (2003) Aberrant actin cytoskeleton leads to accelerated proliferation of 
corneal epithelial cells in mice deficient for destrin (actin depolymerizing factor). Hum Mol Genet 12, 1029-1037

42. Zhao B, Li L, Wang L, Wang CY, Yu J and Guan KL (2012) Cell detachment activates the Hippo pathway via cytoskeleton reorganization to induce anoikis. Genes Dev 26, 54-68

43. Codelia VA, Sun G and Irvine KD (2014) Regulation of YAP by mechanical strain through Jnk and Hippo signaling. Curr Biol 24, 2012-2017

44. Calvo F, Ege N, Grande-Garcia A et al (2013) Mechanotransduction and YAP-dependent matrix remodelling is required for the generation and maintenance of cancerassociated fibroblasts. Nat Cell Biol 15, 637-646

45. Chan SW, Lim CJ, Guo K et al (2008) A role for TAZ in migration, invasion, and tumorigenesis of breast cancer cells. Cancer Res 68, 2592-2598

46. Cordenonsi M, Zanconato F, Azzolin L et al (2011) The Hippo transducer TAZ confers cancer stem cell-related traits on breast cancer cells. Cell 147, 759-772

47. Kim KM, Choi YJ, Hwang JH et al (2014) Shear stress induced by an interstitial level of slow flow increases the osteogenic differentiation of mesenchymal stem cells through TAZ activation. PLoS One 9, e92427

48. Wang KC, Yeh YT, Nguyen P et al (2016) Flow-dependent YAP/TAZ activities regulate endothelial phenotypes and atherosclerosis. Proc Natl Acad Sci U S A 113, 1152511530

49. Sabine A, Bovay E, Demir CS et al (2015) FOXC2 and fluid shear stress stabilize postnatal lymphatic vasculature. J Clin Invest 125, 3861-3877

50. Miller E, Yang J, DeRan M et al (2012) Identification of serum-derived sphingosine-1-phosphate as a small molecule regulator of YAP. Chem Biol 19, 955-962

51. Yu FX, Zhao B, Panupinthu N et al (2012) Regulation of the Hippo-YAP pathway by G-protein-coupled receptor signaling. Cell 150, 780-791

52. Cai H and Xu Y (2013) The role of LPA and YAP signaling in long-term migration of human ovarian cancer cells. Cell Commun Signal 11, 31

53. Mo JS, Yu FX, Gong R, Brown JH and Guan KL (2012) Regulation of the Hippo-YAP pathway by proteaseactivated receptors (PARs). Genes Dev 26, 2138-2143

54. Lappano R and Maggiolini M (2011) G protein-coupled receptors: novel targets for drug discovery in cancer. Nat Rev Drug Discov 10, 47-60

55. Yu FX, Zhang Y, Park HW et al (2013) Protein kinase A activates the Hippo pathway to modulate cell proliferation and differentiation. Genes Dev 27, 1223-1232

56. Zhu $H$, Cheng $X, N i u$ et al (2015) Proton-sensing GPCR-YAP Signalling Promotes Cell Proliferation and Survival. Int J Biol Sci 11, 1181-1189

57. Zhu H, Guo S, Zhang Y et al (2016) Proton-sensing GPCR-YAP Signalling Promotes Cancer-associated Fibroblast Activation of Mesenchymal Stem Cells. Int J Biol Sci 12, 389-396

58. Zhou X, Wang S, Wang Z et al (2015) Estrogen regulates Hippo signaling via GPER in breast cancer. J Clin Invest $125,2123-2135$

59. Feng X, Liu P, Zhou $X$ et al (2016) Thromboxane A2 Activates YAP/TAZ Protein to Induce Vascular Smooth
Muscle Cell Proliferation and Migration. J Biol Chem 291, 18947-18958

60. Wennmann DO, Vollenbroker B, Eckart AK et al (2014) The Hippo pathway is controlled by Angiotensin II signaling and its reactivation induces apoptosis in podocytes. Cell Death Dis 5, e1519

61. Chen D, Sun Y, Wei $Y$ et al (2012) LIFR is a breast cancer metastasis suppressor upstream of the Hippo-YAP pathway and a prognostic marker. Nat Med 18, 15111517

62. Fan R, Kim NG and Gumbiner BM (2013) Regulation of Hippo pathway by mitogenic growth factors via phosphoinositide 3-kinase and phosphoinositide-dependent kinase-1. Proc Natl Acad Sci U S A 110, 2569-2574

63. Azzolin L, Panciera T, Soligo S et al (2014) YAP/TAZ incorporation in the beta-catenin destruction complex orchestrates the Wnt response. Cell 158, 157-170

64. Barry ER, Morikawa T, Butler BL et al (2013) Restriction of intestinal stem cell expansion and the regenerative response by YAP. Nature 493, 106-110

65. Heallen T, Zhang M, Wang J et al (2011) Hippo pathway inhibits Wnt signaling to restrain cardiomyocyte proliferation and heart size. Science 332, 458-461

66. Rosenbluh J, Nijhawan D, Cox AG et al (2012) betaCatenin-driven cancers require a YAP1 transcriptional complex for survival and tumorigenesis. Cell 151, 1457-1473

67. Varelas X, Miller BW, Sopko R et al (2010) The Hippo pathway regulates $\mathrm{Wnt} /$ beta-catenin signaling. Dev Cell 18, 579-591

68. Park HW, Kim YC, Yu B et al (2015) Alternative Wnt Signaling Activates YAP/TAZ. Cell 162, 780-794

69. Azzolin L, Zanconato F, Bresolin S et al (2012) Role of TAZ as mediator of Wnt signaling. Cell 151, 1443-1456

70. Alarcon C, Zaromytidou Al, Xi Q et al (2009) Nuclear CDKs drive Smad transcriptional activation and turnover in BMP and TGF-beta pathways. Cell 139, 757-769

71. Fujii M, Toyoda T, Nakanishi $\mathrm{H}$ et al (2012) TGF-beta synergizes with defects in the Hippo pathway to stimulate human malignant mesothelioma growth. J Exp Med 209, 479-494

72. Varelas X, Sakuma R, Samavarchi-Tehrani P et al (2008) TAZ controls Smad nucleocytoplasmic shuttling and regulates human embryonic stem-cell self-renewal. Nat Cell Biol 10, 837-848

73. Feng X, Degese MS, Iglesias-Bartolome $R$ et al (2014) Hippo-independent activation of YAP by the GNAQ uveal melanoma oncogene through a trio-regulated rho GTPase signaling circuitry. Cancer Cell 25, 831-845

74. Liu G, Yu FX, Kim YC et al (2015) Kaposi sarcomaassociated herpesvirus promotes tumorigenesis by modulating the Hippo pathway. Oncogene 34, 3536-3546

75. Yu FX, Luo J, Mo JS et al (2014) Mutant Gq/11 promote uveal melanoma tumorigenesis by activating YAP. Cancer Cell 25, 822-830

76. DeRan M, Yang J, Shen $\mathrm{CH}$ et al (2014) Energy stress regulates hippo-YAP signaling involving AMPK-mediated regulation of angiomotin-like 1 protein. Cell Rep 9, 495-503

77. Mo JS, Meng Z, Kim YC et al (2015) Cellular energy stress 
induces AMPK-mediated regulation of YAP and the Hippo pathway. Nat Cell Biol 17, 500-510

78. Wang W, Xiao ZD, Li X et al (2015) AMPK modulates Hippo pathway activity to regulate energy homeostasis. Nat Cell Biol 17, 490-499

79. Mohseni M, Sun J, Lau A et al (2014) A genetic screen identifies an LKB1-MARK signalling axis controlling the Hippo-YAP pathway. Nat Cell Biol 16, 108-117

80. Nguyen HB, Babcock JT, Wells CD and Quilliam LA (2013) LKB1 tumor suppressor regulates AMP kinase/mTORindependent cell growth and proliferation via the phosphorylation of Yap. Oncogene 32, 4100-4109

81. Gailite I, Aerne BL and Tapon N (2015) Differential control of Yorkie activity by LKB1/AMPK and the Hippo/ Warts cascade in the central nervous system. Proc Natl Acad Sci U S A 112, E5169-5178

82. Park YY, Sohn BH, Johnson RL et al (2016) Yes-associated protein 1 and transcriptional coactivator with PDZbinding motif activate the mammalian target of rapamycin complex 1 pathway by regulating amino acid transporters in hepatocellular carcinoma. Hepatology 63, 159-172

83. Hansen CG, Ng YL, Lam WL, Plouffe SW and Guan KL (2015) The Hippo pathway effectors YAP and TAZ promote cell growth by modulating amino acid signaling to mTORC1. Cell Res 25, 1299-1313

84. Artinian N, Cloninger C, Holmes B, Benavides-Serrato A, Bashir T and Gera J (2015) Phosphorylation of the Hippo Pathway Component AMOTL2 by the mTORC2 Kinase Promotes YAP Signaling, Resulting in Enhanced Glioblastoma Growth and Invasiveness. J Biol Chem 290, 1938719401

85. Sciarretta S, Zhai $P$, Maejima $Y$ et al (2015) mTORC2 regulates cardiac response to stress by inhibiting MST1. Cell Rep 11, 125-136

86. Liang N, Zhang C, Dill P et al (2014) Regulation of YAP by $\mathrm{mTOR}$ and autophagy reveals a therapeutic target of tuberous sclerosis complex. J Exp Med 211, 2249-2263

87. Parker J and Struhl G (2015) Scaling the Drosophila Wing: TOR-Dependent Target Gene Access by the Hippo Pathway Transducer Yorkie. PLoS Biol 13, e1002274

88. Tumaneng K, Schlegelmilch K, Russell RC et al (2012) YAP mediates crosstalk between the Hippo and PI(3)KTOR pathways by suppressing PTEN via miR-29. Nat Cell Biol 14, 1322-1329

89. Cinar B, Collak FK, Lopez D et al (2011) MST1 is a multifunctional caspase-independent inhibitor of androgenic signaling. Cancer Res 71, 4303-4313

90. Collak FK, Yagiz K, Luthringer DJ, Erkaya B and Cinar B (2012) Threonine- 120 phosphorylation regulated by phosphoinositide-3-kinase/Akt and mammalian target of rapamycin pathway signaling limits the antitumor activity of mammalian sterile 20-like kinase 1. J Biol Chem 287, 23698-23709

91. Garcia-Martinez JM and Alessi DR (2008) mTOR complex 2 (mTORC2) controls hydrophobic motif phosphorylation and activation of serum- and glucocorticoid-induced protein kinase 1 (SGK1). Biochem J 416, 375-385

92. Ikenoue T, Inoki K, Yang Q, Zhou X and Guan KL (2008) Essential function of TORC2 in PKC and Akt turn motif phosphorylation, maturation and signalling. EMBO J 27, 1919-1931

93. Facchinetti V, Ouyang W, Wei $\mathrm{H}$ et al (2008) The mammalian target of rapamycin complex 2 controls folding and stability of Akt and protein kinase C. EMBO J 27, 1932-1943

94. Sorrentino G, Ruggeri N, Specchia V et al (2014) Metabolic control of YAP and TAZ by the mevalonate pathway. Nat Cell Biol 16, 357-366

95. Wehr MC, Holder MV, Gailite I et al (2013) Salt-inducible kinases regulate growth through the Hippo signalling pathway in Drosophila. Nat Cell Biol 15, 61-71 